\title{
Recent results from CMD-2 and SND experiments at VEPP-2M
}

\author{
Simon Eidelman*i \\ Budker Institute of Nuclear Physics, Russia \\ E-mail: eidelman@inp.nsk.su
}

\begin{abstract}
New results on various channels of $e^{+} e^{-}$annihilation into hadrons from CMD-2 and SND experiments at VEPP-2M in Novosibirsk are described. Both detectors collected about $30 \mathrm{pb}^{-1}$ each in the c.m.energy range from $370 \mathrm{MeV}$ to $1380 \mathrm{MeV}$. The main parameters of the $\rho, \omega$ and $\phi$ mesons were determined with high precision. Rare decay modes of the lightest vector mesons were measured with branching fractions $\sim 10^{-4}-10^{-5}$. For the first time radiative processes $e^{+} e^{-} \rightarrow \pi^{0} \gamma$ and $e^{+} e^{-} \rightarrow \eta \gamma$ were studied in the whole energy range. Cross sections of $e^{+} e^{-}$ annihilation into various hadronic final states were measured.
\end{abstract}

International Europhysics Conference on High Energy Physics

July 21st - 27th 2005

Lisboa, Portugal

*Speaker.

${ }^{\dagger}$ For the CMD-2 and SND Collaborations. 
Studies of low energy $e^{+} e^{-}$annihilation into hadrons provide important information on the interactions of light quarks and meson spectroscopy. High precision measurements of the exclusive cross sections and $R$ allow an improvement of accuracy in the calculations of hadronic contributions to the muon anomalous magnetic moment $a_{\mu}$, the value of the fine structure constant $\alpha\left(M_{\mathrm{Z}}^{2}\right)$ and hyperfine splitting in Muonium [1].

Since 1974 the VEPP-2M $e^{+} e^{-}$collider has been successfully running in the Budker Institute of Nuclear Physics in Novosibirsk in the c.m.energy range from the threshold of hadron production to $1400 \mathrm{MeV}$ [2]. Its maximum luminosity reached $\sim 3 \cdot 10^{30} \mathrm{~cm}^{-2} \mathrm{~s}^{-1}$ at the $\phi$ meson energy. In the last series of experiments two detectors, CMD-2 [3] and SND [4], collected about $30 \mathrm{pb}^{-1}$ each from $370 \mathrm{MeV}$ to $1380 \mathrm{MeV}$.

Both groups measured the cross section of the process $e^{+} e^{-} \rightarrow \pi^{+} \pi^{-}$in the energy range of the $\rho$ meson. These results can be used to calculate the leading order hadronic contribution to $a_{\mu}$ shown in Table 1(a) together with that of KLOE [5]. Although the SND result is somewhat higher, they are all compatible within current experimental errors. From a fit of their data shown in Fig. 1(a) the SND group determined the $\rho$ and $\omega$ meson parameters compared in Table 1(b) to the world average data.

\begin{tabular}{|c|c|}
\hline Group & $a_{\mu}, 10^{-10}$ \\
\hline CMD-2 & $378.6 \pm 3.6$ \\
\hline KLOE & $375.6 \pm 5.0$ \\
\hline SND & $385.6 \pm 5.2$ \\
\hline
\end{tabular}

(a) $a_{\mu}, 600 \mathrm{MeV}<\sqrt{s}<960 \mathrm{MeV}$

\begin{tabular}{|c|c|c|}
\hline Parameter & SND & PDG-2005 \\
\hline$M_{\rho}, \mathrm{MeV}$ & $774.9 \pm 0.6$ & $775.8 \pm 0.5$ \\
\hline$\Gamma_{\rho}, \mathrm{MeV}$ & $146.5 \pm 1.7$ & $146.4 \pm 1.5$ \\
\hline $\mathscr{B}\left(\omega \rightarrow \pi^{+} \pi^{-}\right), \%$ & $1.75 \pm 0.11$ & $1.70 \pm 0.27$ \\
\hline
\end{tabular}

(b) $\rho-\omega$ Parameters

Table 1: Results in the $\rho$ meson region

SND has completed its analysis of the annihilation into $\pi^{0} \gamma$ and $\eta \gamma$. Figure 1(b) shows the cross section of the process $e^{+} e^{-} \rightarrow \pi^{0} \gamma$ while in Table 2 their results are compared to the previous world best coming from the CMD-2 detector [6]. SND results are consistent with those from CMD-2 and have higher accuracy.

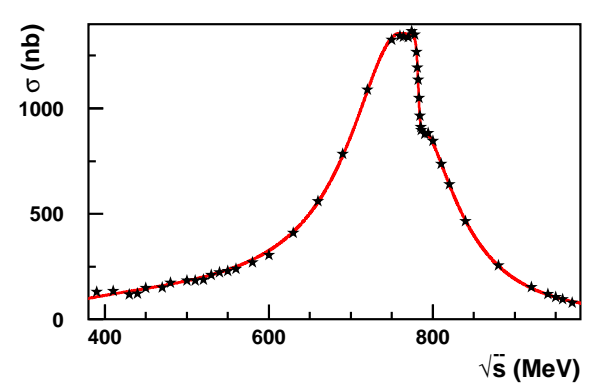

(a) $e^{+} e^{-} \rightarrow \pi^{+} \pi^{-}$at SND

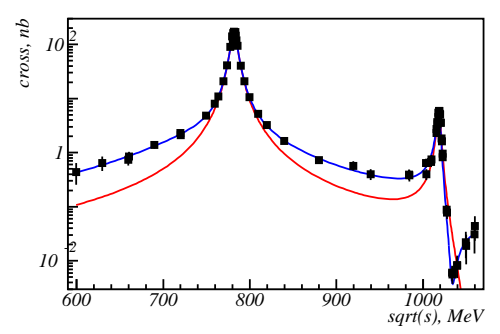

(b) $e^{+} e^{-} \rightarrow \pi^{0} \gamma$ at SND

Figure 1: SND results on $e^{+} e^{-} \rightarrow \pi^{+} \pi^{-}$and $e^{+} e^{-} \rightarrow \pi^{0} \gamma$

SND is continuing the analysis of the annihilation into kaon pairs. Results on the charged kaons demonstrated in Fig. 2(a) show that the SND data are above those from OLYA and that there is no evidence for the $\phi^{\prime}$. In contrast to this finding, in the $K_{S}^{0} K_{L}^{0}$ mode shown in Fig. 2(b) SND data are consistent with those from CMD-2 and evidence for the $\phi^{\prime}$ has been obtained. 


\begin{tabular}{|c|c|c|c|c|c|}
\hline Meson & SND & CMD-2 & Meson & SND & CMD-2 \\
\hline$\rho, 10^{-4}$ & $5.02 \pm 0.73$ & $6.71 \pm 1.34$ & $\rho, 10^{-4}$ & $2.77 \pm 0.31$ & $3.39 \pm 0.48$ \\
\hline$\omega, 10^{-2}$ & $8.49 \pm 0.26$ & $9.01 \pm 0.59$ & $\omega, 10^{-4}$ & $4.22 \pm 0.50$ & $4.75 \pm 0.78$ \\
\hline$\phi, 10^{-3}$ & $1.36 \pm 0.10$ & $1.26 \pm 0.10$ & $\phi, 10^{-2}$ & $1.341 \pm 0.052$ & $1.287 \pm 0.064$ \\
\hline
\end{tabular}

Branching fractions $\mathscr{B}\left(V \rightarrow \pi^{0} \gamma\right) \quad$ Branching fractions $\mathscr{B}(V \rightarrow \eta \gamma)$

Table 2: SND and CMD-2 results on $e^{+} e^{-} \rightarrow \pi^{0} \gamma, \eta \gamma$

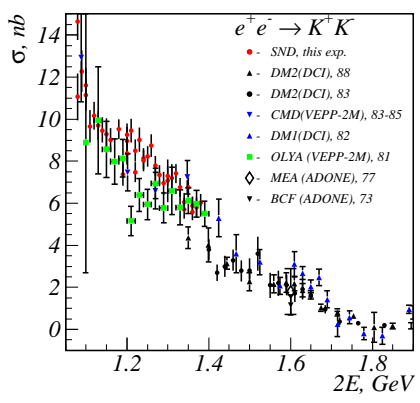

(a) $e^{+} e^{-} \rightarrow K^{+} K^{-}$

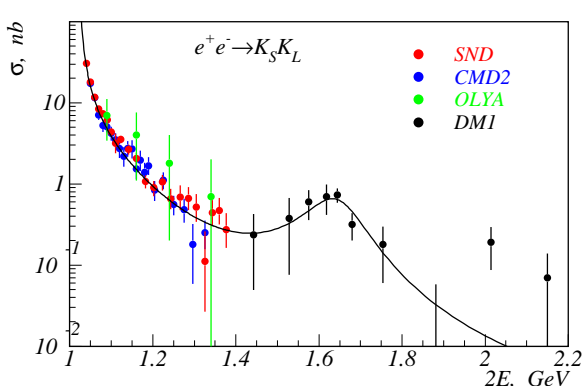

(b) $e^{+} e^{-} \rightarrow K_{S}^{0} K_{L}^{0}$

Figure 2: SND results on $e^{+} e^{-} \rightarrow K^{+} K^{-}, K_{S}^{0} K_{L}^{0}$

CMD-2 made a simultaneous analysis of the four main decay modes of the $\phi$ meson. A combined fit of the data allows to obtain in a single experiment the accuracy of its main parameters close to the world average one [7], see Table 3. They also measured the energy dependence of the ratio of the $K^{+} K^{-}$and $K_{S}^{0} K_{L}^{0}$ yields predicted by Ref. [8].

\begin{tabular}{|c|c|c|}
\hline Quantity & CMD-2 & PDG-2005 \\
\hline$M_{\phi}, \mathrm{MeV}$ & $1019.444 \pm 0.026$ & $1019.456 \pm 0.020$ \\
\hline$\Gamma_{\phi}, \mathrm{MeV}$ & $4.256 \pm 0.031$ & $4.26 \pm 0.05$ \\
\hline $\mathscr{B}\left(\phi \rightarrow e^{+} e^{-}\right), 10^{-4}$ & $2.86 \pm 0.05$ & $2.98 \pm 0.04$ \\
\hline
\end{tabular}

Table 3: CMD-2 results on the $\phi$ meson parameters

Analysis of the process $e^{+} e^{-} \rightarrow \pi^{+} \pi^{-} \pi^{0} \pi^{0}$ by CMD-2 (Fig. 3) allowed to conclude that it is dominated by $a_{1} \pi$ and $\omega \pi$ and due to their interference $\sigma_{\pi^{+} \pi^{-} 2 \pi^{0}} \neq \sigma_{a_{1} \pi}+\sigma_{\omega \pi}$. It is also observed that separation of $a_{1} \pi$ and $\omega \pi$ differs in CMD-2 and SND [9].

CMD-2 performed analysis of the reaction $e^{+} e^{-} \rightarrow \pi^{+} \pi^{-} \pi^{0}$ and concluded that $m_{\pi \pi}$ spectra show $\rho \pi$ dominance with an $\omega \pi$ admixture; a combined fit of SND [10], CMD-2 and BaBar [11] data is consistent with two higher $\omega$ states [7]; the cross sections obtained by DM2 [12] are signif-
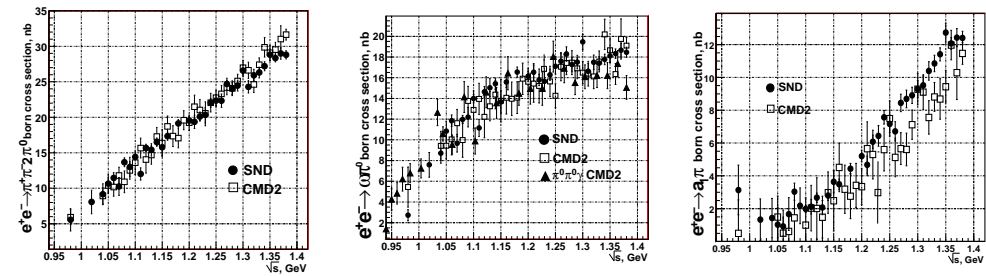

Figure 3: CMD-2 results on $e^{+} e^{-} \rightarrow \pi^{+} \pi^{-} \pi^{0} \pi^{0}$ 


\begin{tabular}{|l|c|c|c|c|}
\hline Decay & CMD-2 & SND & RPP-2004 & Theory \\
\hline$\omega \rightarrow \pi^{0} e^{+} e^{-}$ & $8.19 \pm 0.71 \pm 0.62$ & $8.09 \pm 0.53 \pm 0.79$ & $5.9 \pm 1.9$ & $7.2-8.0$ \\
\hline$\omega \rightarrow \eta e^{+} e^{-}$ & $<0.11$ & - & - & $0.020-0.048$ \\
\hline$\rho \rightarrow \pi^{0} e^{+} e^{-}$ & $<0.16$ & - & - & $0.041-0.065$ \\
\hline$\rho \rightarrow \eta e^{+} e^{-}$ & $<0.07$ & - & - & $0.022-0.032$ \\
\hline
\end{tabular}

Table 4: Branching fractions of conversion decays, $10^{-4}$

icantly lower than those of $\mathrm{BaBar}$.

Table 4 shows results of a study of conversion decays of the $\rho$ and $\omega$ mesons, which provide a further test of the Vector Dominance Model and allow a measurement of transition form factors. In addition, precise knowledge of their branching ratios is necessary to estimate the background in searches for quark-gluon plasma.

In conclusion, we reported a high precision measurement of the main parameters of the $\rho, \omega$, $\phi$, which showed validity of the Vector Dominance Model. Various rare decay modes of the light vector mesons were studied with a sensitivity to $\mathscr{B} \sim 10^{-5}-10^{-6}$. Exclusive hadronic final states were measured with low systematic errors allowing significant improvement in $a_{\mu}^{\text {had,LO }}$.

Future progress is expected from experiments at the upgraded collider VEPP-2000 [13] with the maximum center-of-mass energy $2 \mathrm{GeV}$ and luminosity $10^{32} \mathrm{~cm}^{-2} \mathrm{~s}^{-1}$.

I'm grateful to my colleagues from CMD-2 and SND and particularly to M.N. Achasov, T.V. Dimova, D.A. Gorbachev, V.F. Kazanin, A.A. Korol, I.B. Logashenko, P.A. Lukin and S.I. Serednyakov for their help. This work was supported in part by the grants RFBR-03-02-16843 and DFG GZ: 436 Rus113/769/0-1R.

\section{References}

[1] S. Eidelman and F. Jegerlehner, Z. Phys. C 67, 585 (1995); A. Czarnecki, S.I. Eidelman and S.G. Karshenboim, Phys Rev. D 65, 053004 (2002).

[2] V.V. Anashin et al., Preprint INP 84-114, Novosibirsk, 1984.

[3] E.V. Anashkin et al., ICFA Instr. Bull. 5, 18 (1988).

[4] M.N. Achasov et al., Preprint BudkerINP 96-47, Novosibirsk, 1996.

[5] A. Aloisio et al., Phys. Lett. B 606, 12 (2005).

[6] R.R. Akhmetshin et al., Phys. Lett. B 509, 217 (2001); ibid B 605, 26 (2005).

[7] S. Eidelman et al., Phys. Lett. B 592, 1 (2004).

[8] M.B.Voloshin, Sov. J. Nucl. Phys. 68, 771 (2005).

[9] M.N. Achasov et al., J. Exp. Theor. Phys. 96, 789 (2003).

[10] M.N. Achasov et al., Phys. Rev.. D 66, 032001 (2002).

[11] B. Aubert et al., Phys. Rev. D 70, 072004 (2004).

[12] A. Antonelli et al., Zeit. f. Phys. C 56, 15 (1992).

[13] Yu.M. Shatunov et al., Proc. of the VIIth Eur. Part.Accel. Conf., Vienna 2000, EPAC (2000), p.439. 